\begin{tabular}{|c|c|c|c|}
\hline$\gamma \cdot 10^{4}$ & $\Theta^{*}$ & $\begin{array}{l}\text { Tempe- } \\
\text { rature } \\
\text { range, } \\
{ }^{\circ} \mathrm{K}\end{array}$ & Authors \\
\hline 32,9 & - & $1,8-4,2$ & Guthrie, Friedberg, \\
\hline 43 & - & $1,2-20$ & WOLCOTT ${ }^{3}$ \\
\hline 28 & 392 & $11-20$ & $\begin{array}{l}\text { BoOTH, HOARE, and } \\
\text { MURPHY } 4\end{array}$ \\
\hline $35-40$ & - & $14-22$ & $\begin{array}{l}\text { ARMSTRONG and } \\
\text { GRAYSON-SMITH }\end{array}$ \\
\hline 42,1 & 410 & $16-22$ & $\begin{array}{l}\text { Elson, Grayson-SMith, } \\
\text { and Wilhelm }{ }^{6}\end{array}$ \\
\hline 32,8 & 415 & $10-21$ & this paper \\
\hline
\end{tabular}

Table 6. Values of the electronic heat coefficient, $\gamma$ (cal/ $\mathrm{deg}^{2} \cdot \mathrm{g}$-atom), for alpha-manganese.

3. Entropies at $25{ }^{\circ} \mathrm{C}$ have been calculated graphically as: $7,6_{5} \mathrm{cal} / \mathrm{deg} \cdot \mathrm{g}$-atom for alpha-manganese and $7,9_{8} \mathrm{cal} / \mathrm{deg} \cdot \mathrm{g}$-atom for gamma-manganese.

Kelley, on the basis of his own measurements between $\sim 50{ }^{\circ} \mathrm{K}$ and room temperature, calculated an entropy value of $7,61 \pm 0,06$, while Shomate, using his own data in the same temperature range, gave $7,59 \pm 0,04 \mathrm{cal} / \mathrm{deg} \cdot \mathrm{g}$-atom. Both these values are consistent with the one we have found for $\alpha$-Mn.

As to gamma-manganese, our figure may be compared only with the one (remarkably lower) of Shomate, who calculated $7,72 \pm 0,04 \mathrm{cal} / \mathrm{deg} \cdot \mathrm{g}$-atom.

The authors are indebted to Dr. O. WinkLer of the Firm Balzers Gerätebauanstalt (Balzers, Fürstentum Liechtenstein) for having manufactured the samples, to the Deutsche Forschungsgemeinschaft for having supplied a precision bridge for resistance measurements, and to the Schweizerischen Nationalfonds for having financially supported the present research.

P. Franzosini and C. G. Losa heartily thank Dr. U. Piesbergen, Oberassistent at the Institute of Physical Chemistry (Zurich, Switzerland), for the precious help he has given them in the drawing up of the manuscript after the death of Prof. Dr. K. Cuusius.

\title{
Polarisationsoptische Temperaturmessung und Filterung bei Rubin-Laser
}

\author{
Von Hans-Georg HäFele \\ OSRAM GmbH Studiengesellschaft, Augsburg \\ (Z. Naturforschg. 19 a, 1353-1356 [1964] ; eingegangen am 21. August 1964)
}

\begin{abstract}
Die Temperatur im Inneren eines homogenen Laser-Rubins läßt sich auf Grund seiner Doppelbrechung auf optischem Wege ermitteln, indem man ihn zwischen zwei gekreuzte Polarisatoren bringt und die spektrale Lage der Polarisations-Interferenzstreifen bestimmt. Im Gebiet der R-LinienFluoreszenz verschieben sich die Interferenzstreifen zwischen 18 und $32{ }^{\circ} \mathrm{C}$ mit der Temperatur nach kürzeren Wellenlängen; es ist $\Delta \lambda / \Delta T=-0,83 \AA$ /grad. Bei der Anregung eines Rubins mit einer stabförmigen Xe-Blitzröhre in einem elliptischen Reflektor erhöht sich im vorliegenden Fall die Temperatur beim Schwellenwert um $1,1^{\circ} \mathrm{C}$. Bei Verwendung einer wendelförmigen Röhre wird der Kristall um $3,1^{\circ} \mathrm{C}$ erwärmt. Während bei der stabförmigen Röhre die Temperatur nach der Anregung im Laufe von ca. 10 min monoton auf die Umgebungstemperatur absinkt, nimmt sie bei der Wendellampe infolge Wärmeleitung von der heißen Quarzwendel zunächst beträchtlich zu. Mit Hilfe eines aus einer Kristallplatte und zwei GLAN-Tномson-Prismen bestehenden einfachen PolarisationsInterferenz-Filters läßt sich die Güte eines Laser-Resonators auch in sehr eng benachbarten Spektralbereichen beliebig variieren.
\end{abstract}

Bei der Anregung von Festkörper-Lasern wird im Kristall ein Teil der Pumpstrahlung in Wärme umgewandelt. Im Grundgitter werden Schwingungen angeregt, die nach kurzer Zeit im thermodynamischen Gleichgewicht sind und eine Temperaturerhöhung hervorrufen. Auch durch die fluoreszierenden Leuchtzentren wird auf Grund des Pumpmechanismus stets Energie an das Gitter übertragen. Beim Rubin gehen z. B. die durch Lichtabsorption in den ${ }^{4} \mathrm{~F}_{1}$ - und ${ }^{4} \mathrm{~F}_{2}$ Banden angeregten $\mathrm{Cr}^{3^{+}}$-Ionen strahlungslos in die beiden ${ }^{2} \mathrm{E}$-Zustände über, wobei die Differenz der
Anregungsenergie vom Gitter aufgenommen wird. Die Kopplung der Leuchtzentren an das Gitter zeigt sich bekanntlich auch in der Überlagerung von Schwingungs- und Elektronenübergängen, die man in den Absorptions- und Fluoreszenzspektren des Rubins und anderer Chromphosphore beobachtet ${ }^{1}$.

Die Temperaturerhöhung bei einem Laser-Kristall hat verschiedenartige Konsequenzen. Einmal wird die Linienbreite mit steigender Temperatur vergrößert und die Quantenausbeute verringert, wodurch die Schwellenenergie für erzwungene Emission

1 E. FIck u. G. Joos, Handbuch der Physik, Bd. 28, Spektroskopie II, Springer-Verlag, Berlin 1957. 
ansteigt ${ }^{2}$. Zum anderen verändert die Temperatur die Länge und den Brechungsindex des Kristalls und beeinflußt dadurch die Eigenschwingungen des optischen Resonators. An zeitlich aufgelösten PerotFABRY-Aufnahmen der Rubin-Laser-Strahlung sieht man oft, daß in aufeinanderfolgenden Einzelimpulsen (Spikes) verschiedene Eigenfrequenzen des Resonators auftreten, was auf die Temperaturerhöhung des Kristalls während der Anregung zurückzuführen ist $^{3}$.

Erfolgt die Lichteinstrahlung unsymmetrisch, so entstehen darüber hinaus lokale Temperaturgradienten, die ihrerseits mechanische Spannungen verursachen. Solche Inhomogenitäten konnten durch interferometrische Messungen beobachtet werden ${ }^{4}$.

Im folgenden wird über Untersuchungen an einem Rubin-Laser berichtet, aus denen sich die Temperaturerhöhungen des Kristalls unter verschiedenen Anregungsbedingungen ergeben.

\section{Meßmethode und Meßanordnung}

Temperaturmessungen im Inneren eines homogenen Kristalls sind mit üblichen Methoden schwer durchzuführen. Es wird daher eine indirekte Methode verwendet, bei welcher die Temperaturabhängigkeit der Doppelbrechung des Rubins ausgenutzt wird.

Durchstrahlt man eine planparallele Platte, die parallel zur optischen Achse aus einem optisch einachsigen Kristall geschnitten ist und sich zwischen zwei gekreuzten (oder parallelen) Polarisatoren befindet, mit kontinuierlichem Licht. so besteht das Durchlässigkeits-Spektrum bekanntlich aus abwechselnd hellen und dunklen Streifen (den sog. Müllerschen Streifen). Die spektrale Lage dieser Polarisations-Interferenzstreifen hängt von der Schichtdicke $d$ und der Doppelbrechung $\mu=n_{0}-n_{\text {ао }}$ des Kristalls ab. Bei gekreuzten Polarisatoren ist

$$
\lambda_{m}=d \mu / m \text {. }
$$

Dabei ist $\lambda_{m}$ die Wellenlänge eines dunklen Streifens mit der Ordnungszahl $m$. Der spektrale Abstand $\lambda_{m}-\lambda_{m \pm 1}$ zweier aufeinanderfolgender Durchlässigkeitsminima ist $\Delta \lambda=\lambda_{m}{ }^{2} / d \mu$. Da sowohl die Doppelbrechung als auch die Schichtdicke von der Temperatur abhängen, kann man aus der spektralen Lage der

2 J. P. W ittke, J. Appl. Phys. 33, 2333 [1962].

3 G. R. Hanes u. B. P. Stoicheff, Nature, Lond. 195, 587 [1962].
Interferenzstreifen die Temperatur im Inneren des Kristalls bestimmen.

Die Methode hat den Vorteil, daß man die mittlere Temperatur des ganzen Volumens erfaßt, und daß der Kristall in der Laser-Anordnung nicht verändert wird. Die Bedingungen bei der Temperaturmessung sind also genau dieselben wie beim LaserBetrieb. Es werden lediglich die äußeren Resonatorspiegel entfernt und durch zwei Polarisatoren ersetzt. Temperaturunterschiede innerhalb des Rubins können sich u. U. durch eine Krümmung der Interferenzstreifen bemerkbar machen, die man allerdings bei dem vorhandenen Auflösungsvermögen nicht beobachtet.

Die Messungen werden hier an einem Rubin $(0,04 \%$ $\mathrm{Cr}$ ) von $7,0 \mathrm{~mm}$ Dicke und $59,75 \mathrm{~mm}$ Länge durchgeführt. Seine Längsachse verläuft senkrecht zur optischen Achse, die beiden Endflächen sind nicht verspiegelt. Die optische Achse bildet Winkel von jeweils $45^{\circ}$ mit den beiden gekreuzten Polarisatoren $\mathrm{P}$ und $\mathrm{A}$, die links und rechts vom Rubin in den Strahlengang gebracht

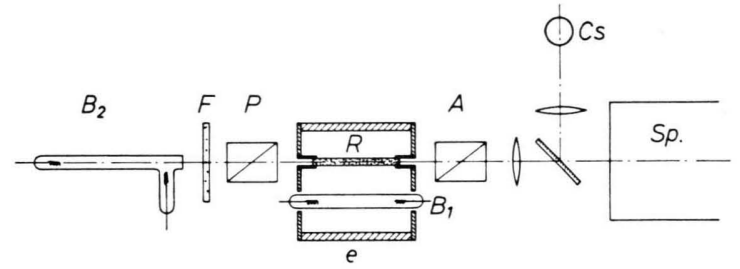

Abb. 1. Schematische Darstellung der Meßanordnung. $\mathrm{R}=$ Rubin $; \mathrm{e}=$ elliptischer Reflektorspiegel $; \mathrm{P}, \mathrm{A}=\mathrm{G}_{\mathrm{LAN}-}$ Thомsом-Prismen; $\mathrm{B}_{1}=$ stabförmige Blitzröhre OSRAM Bl 5159; $\mathrm{B}_{2}=$ abgewinkelte Blitzröhre, Variante der OSRAM B1 6150 mit Planfenster (Blitzenergie bei allen Aufnahmen $200 \mathrm{Ws}) ; \mathrm{F}=$ Filter $; \mathrm{Sp}=$ Steinheil-Dreiprismen-Spektralapparat (Dispersion $59,5 \AA / \mathrm{mm}$ bei $6973 \AA$ ) ; Cs = Cs-Spektrallampe zur Wellenlängeneichung.

werden (Abb. 1). Für die Anregung des Kristalls wird sowohl eine stabförmige Xe-Blitzröhre OSRAM B1 5159 in Verbindung mit einem elliptischen Reflektor, als auch eine wendelförmige Blitzröhre verwendet. Die Meßstrahlung liefert eine zweite Blitzröhre, die eine Blitzdauer von nur etwa $100 \mu \mathrm{sec}$ besitzt. Es handelt sich um eine Variante der Xe-Hochleistungsblitzröhre OSRAM B1 6150, die für diesen Zweck in einer abgewinkelten Form (End-on-Form) gebaut wurde. Die Röhre ist an der Stirnseite mit einer Planscheibe abgeschlossen, durch welche das Blitzlicht als homogenes Bündel austreten kann. Ein S c h o t t - Glasfilter RG 5 sorgt dafür, daß die Meßstrahlung nur Wellenlängen zwischen etwa 650 und $2700 \mathrm{~nm}$ enthält, welche vom Rubin, abgesehen von den relativ schwachen R-Linien, nicht absorbiert werden.

4 A. E. Blume u. K. F. Tittel, Appl. Optics 3, 527 [1964]. 
Die Temperaturabhängigkeit der Doppelbrechung vom Rubin ist nicht bekannt. Man erhält sie aus der Verschiebung der Polarisations-Interferenzstreifen, wobei man den Kristall in einer Wasserküvette mit Hilfe eines Ultrathermostaten auf verschiedene Temperaturen bringt.

\section{Meßergebnisse}

a) Zwei typische Streifenspektren für etwas verschiedene Temperaturen sind in Abb. 2 wiedergegeben. Links von der starken Cs-Linie bei $6973,3 \AA$ erkennt man die beiden Absorptionslinien $R_{1}$ und $\mathrm{R}_{2}$ des Rubins bei 6943 und $6929 \AA$. Der Wellenlängenabstand zwischen zwei aufeinanderfolgenden Minima beträgt $9,1 \AA$. Die Doppelbrechung ergibt sich in diesem Bereich (genau bei $6973 \AA$ ) zu

$$
\mu=0,00894 \text {. }
$$

Dieser Wert ist etwas größer als beim ungefärbten a-Korund ${ }^{5}$. Die Durchlässigkeitsmaxima und -minima

a)

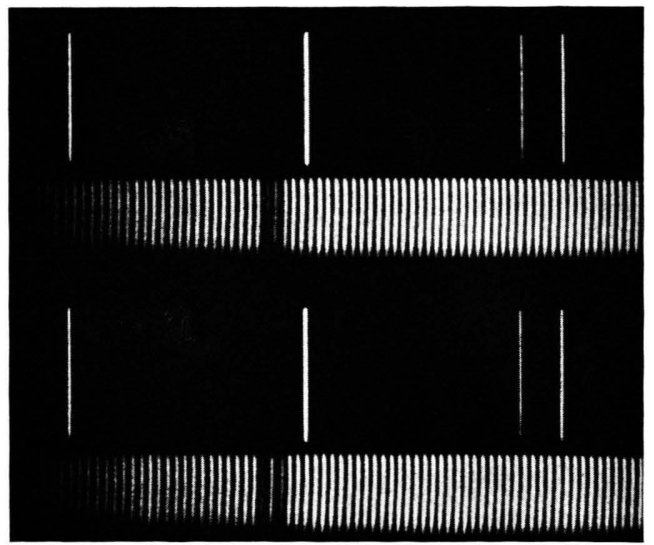

$\lambda \rightarrow$

Abb. 2. Absorptionsspektrum des Rubins zwischen gekreuzten Polarisatoren mit Cs-Eichspektrum. a) vor der Anregung, b) 1 sec nach der Anregung mit 720 Ws. Bei den ausgewerteten Spektren wurde das Cs-Spektrum in das Interferenzstreifensystem hineinphotographiert, wodurch sich die Auswertgenauigkeit erhöht. Während der Dauer der Pumpstrahlung wird der Spalt abgedeckt, da sonst das Spektrum vom Fluoreszenzlicht überstrahlt wird.

verschieben sich im Temperaturbereich zwischen 18 und $32{ }^{\circ} \mathrm{C}$ linear mit der Temperatur nach kürzeren Wellenlängen. Es ist

$$
\Delta \lambda / \Delta T=-0,83[\AA / \mathrm{grad}] .
$$

5 M. A. Jeppesen, J. Opt. Soc. Amer. 48, 629 [1958].
Dieser empirische Wert wird den späteren Temperaturmessungen zugrunde gelegt. Die relative Wellenlängenverschiebung mit der Temperatur setzt sich aus der relativen Änderung des Berechnungsindex und der relativen Längenänderung des Kristalls zusammen:

$$
\frac{\Delta \lambda}{\lambda}=\frac{\Delta \mu}{\mu}+\frac{\Delta d}{d} .
$$

Mit dem Literaturwert $\alpha=5,0 \cdot 10^{-6}\left[\operatorname{grad}^{-1}\right]$ für die lineare Wärmeausdehnung in Richtung senkrecht zur optischen Achse errechnet man als Temperaturabhängigkeit der Doppelbrechung

$$
\Delta \mu / \Delta T=-1,11 \cdot 10^{-6}\left[\operatorname{grad}^{-1}\right] .
$$

Die Verschiebung der Müllerschen Streifen entsteht demnach hauptsächlich durch die Abnahme der Doppelbrechung $\mu$ mit der Temperatur. Die thermische Ausdehnung spielt keine große Rolle.

b) Die Temperaturerhöhungen des Kristalls bei Anregung mit einer wendelförmigen Xe-Blitzröhre bzw. einer stabförmigen Röhre in einem elliptischen Reflektor sind in Abb. 3 in Abhängigkeit von der

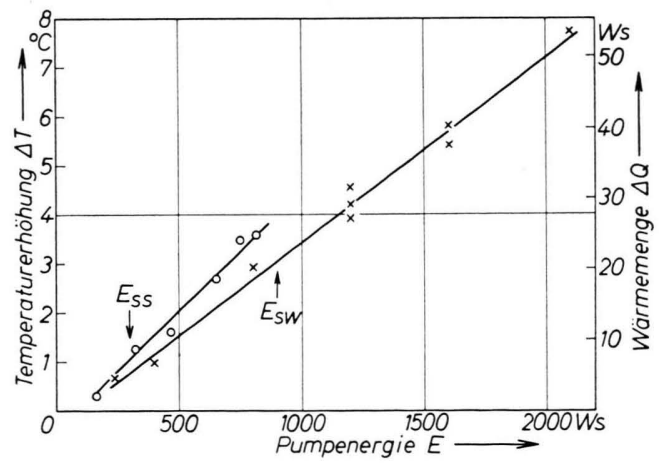

Abb. 3. Erhöhung von Temperatur und Wärmemenge des Laser-Kristalls durch die Pumpstrahlung einer wendelförmigen $\left(x_{-} \times-\times-\right)$und einer stabförmigen $\left(\mathrm{O}_{-} \mathrm{O}-\mathrm{O}-\right) \mathrm{Xe}$ Blitzröhre. $E_{\mathrm{SW}}=$ Schwellenenergie bei Anregung mit der Wendelröhre, $E_{\mathrm{SS}}=$ Schwellenenergie bei der stabförmigen Röhre.

Blitzenergie eingetragen. Als Fehlergrenze sind $0,3{ }^{\circ} \mathrm{C}$ einzusetzen. Sie entsteht durch die Meßunsicherheit von ca. 0,004 $\mathrm{mm}$ beim Vermessen der Streifensysteme. Der Zeitabstand zwischen der Anregung des Kristalls und der Zündung der Meßstrahl-Röhre beträgt bei diesen Aufnahmen einheitlich 1 sec. Die maximale Belastbarkeit liegt bei der stabförmigen Röhre bei ca. $800 \mathrm{Ws}$, bei der Wendelröhre bei ca. $2500 \mathrm{Ws}$. Die Schwellenenergien für die Laser-Schwingungen bei Verwendung von plan- 
parallelen Resonatorspiegeln aus Gold sind in dem Diagramm eingezeichnet. Man sieht, daß der Kristall bei der Anregung dicht über dem Schwellenwert im einen Fall um etwa $3,1^{\circ} \mathrm{C}$, im anderen um etwa $1,1{ }^{\circ} \mathrm{C}$ erwärmt wird.

Aus der Temperaturerhöhung läßt sich unter $\mathrm{Zu}$ grundelegen der spezifischen Wärme von 79 [Ws pro Mol·grad] die zusätzliche Wärmeenergie des Kristalls berechnen. Die Werte sind auf der rechten Skala in Abb. 3 angegeben. Im Kristall werden demnach $2-3 \%$ der elektrischen Energie der Xenon-Entladung in Wärme umgewandelt.

c) Damit man sieht, nach welcher Zeit der Kristall unter den vorhandenen Wärmeleitungsverhältnissen, ohne zusätzliche Kühlung, seine Ausgangstemperatur wieder erreicht hat, verändert man bei konstanter Anregungsenergie den Zeitabstand zwischen der Anregung und dem Meßimpuls. Die dabei gefundenen Temperaturdifferenzen sind in Abb. 4 dargestellt.

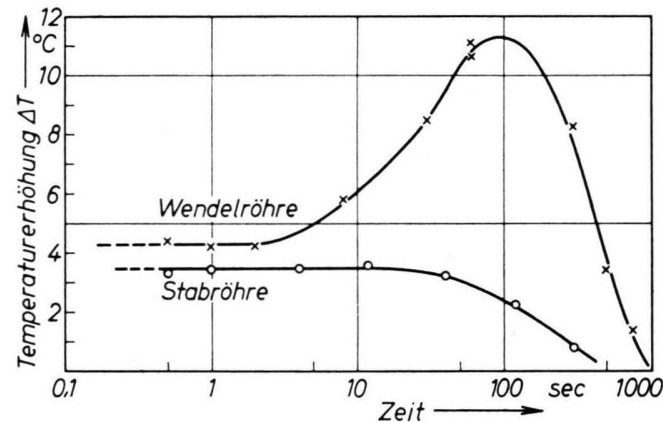

Abb. 4. Zeitlicher Temperaturverlauf des Kristalls nach Anregung mit einer wendelförmigen und einer stabförmigen Blitzröhre. Blitzenergien $1200 \mathrm{Ws}$ bzw. $750 \mathrm{Ws}$.

Bei der wendelförmigen Röhre steigt die Temperatur einige Zeit nach der Anregung noch weiter an und erreicht ein Maximum. Erst dann fällt sie allmählich ab, um nach ca. 15 min ihren Ausgangspunkt zu erreichen. Dieser Temperaturanstieg ist durch den Wärmeübergang von der umgebenden heißen Quarzwendel auf den Kristall zu erklären.

Beim Pumpen mit der stabförmigen Röhre nimmt die Temperatur nach der Anregung monoton ab. Diese Röhre ist räumlich genügend weit vom Kristall getrennt, daher spielt die Wärmeleitung von der

${ }^{6}$ M. HAase, Zeiss-Nachr., 4. Folge 1941, S. $51-57$.

7 F. J. McClung, S. E. Schwarz, R.W. Hellwarth, I. J. D'Haenens u. F. J. Meyers, Bull. Amer. Phys. Soc., Ser. II, 6, 511 [1961].
Röhre zum Kristall keine Rolle. Bei der vorliegenden Anordnung hat der Kristall nach ca. $10 \mathrm{~min}$ seine Umgebungstemperatur wieder erreicht.

\section{Filterung in Laser-Resonatoren mit Polarisations-Interferenzfiltern}

Bekanntlich kann die Auslöschung bestimmter Spektralgebiete nach dem eingangs beschriebenen Prinzip zur Herstellung sehr schmalbandiger Filter verwendet werden ${ }^{6}$. In der Laser-Technik ist es häufig erwünscht, die Resonatorgüte für bestimmte schmale Spektralgebiete sehr hoch, für andere, oft eng benachbarte Spektralgebiete, sehr niedrig zu machen. Dies läßt sich leicht erreichen, wenn man in den Resonator zusätzlich eine zwischen zwei Polarisatoren (z. B. Glan-Thomson-Prismen) angeordnete Kristallplatte einbringt. Durch geeignete Wahl des Kristallmaterials und der wirksamen Schichtdicke können die Spektralgebiete maximaler und minimaler Durchlässigkeit beliebig gelegt werden.

Um z. B. einen Rubin-Laser in der $R_{2}$-Fluoreszenz zu betreiben ${ }^{7}$, muß ein Durchlässigkeitsmaximum auf die Wellenlänge $\lambda_{2}\left(R_{2}\right)=6929 \AA$ und ein Durchlässigkeitsminimum auf $\lambda_{1}\left(R_{1}\right)=6943 \AA$ fallen. Ist diese Bedingung etwa bei gekreuzten Polarisationen erfüllt, so werden bei Drehung auf Parallelstellung die Maxima und Minima gerade vertauscht, so daß die $R_{1}$-Strahlung verstärkt werden kann.

Zur Berechnung der Kristallplatte ergeben sich folgende Bedingungen:

$$
\begin{gathered}
\lambda_{1}=d \mu / m, \quad \lambda_{2}=d \mu /\left(m+\frac{1}{2}\right) \\
d=\frac{\lambda_{1} \lambda_{2}}{2 \mu\left(\lambda_{1}-\lambda_{2}\right)} .
\end{gathered}
$$

Eine Kalkspatplatte mit einer Doppelbrechung $\mu=$ 0,1684 muß beispielsweise $1,020 \mathrm{~mm}$ dick sein, damit der Resonator. wahlweise für die $R_{1}$ - oder $R_{2}$ Linie gesperrt ist. Im Sinne der vorigen Untersuchung lassen sich die Durchlässigkeitsbereiche natürlich auch durch Variation der Kristalltemperatur verschieben. Ferner können piezoelektrische Kristalle benützt werden, deren Doppelbrechungen durch elektrische Felder zu steuern sind ${ }^{8}$.

8 B. H. Billings, J. Opt. Soc. Amer. 39, 797, 802 [1949]. 